\title{
BLOW-UP OF STRAIGHTENING-CLOSED IDEALS IN ORDINAL HODGE ALGEBRAS
}

\author{
WINFRIED BRUNS, ARON SIMIS, AND NGÔ VIÊT TRUNG
}

\begin{abstract}
We study a class of ideals $I$ in graded ordinal Hodge algebras $A$. These ideals are distinguished by the fact that their powers have a canonical standard basis. This leads to Hodge algebra structures on the Rees ring and the associated graded ring. Furthermore, from a natural standard filtration one obtains a depth bound for $A / I^{n}$ which, under certain conditions, is sharp for $n$ large. Frequently one observes that $I^{n}=I^{(n)}$. Under suitable hypotheses it is possible to calculate the divisor class group of the Rees algebra. Our main examples are ideals of "virtual" maximal minors and ideals of maximal minors "fixing a submatrix".
\end{abstract}

\section{INTRODUCTION}

Let $B$ be a commutative (noetherian) ring and let $R$ be a graded ordinal Hodge algebra over $B$ (see $\S 2$ for definitions). Let $H$ denote the underlying poset of $R$. The main object of the present work is to thoroughly study a straightening-closed ideal $\Omega$, i.e., an ideal $\Omega \subset H$ such that, for any incomparable elements $x, y \in \Omega$, if $x y=\sum_{M} b_{M} M$ is the corresponding straightening, then every $M$ contains at least two factors belonging to $\Omega$.

This concept was first introduced by Huneke, who showed that if $\Omega$ is a straightening-closed ideal then the elements of $\Omega$ form a weak $d$-sequence in $R$ [Hu 2]. From an analysis of such sequences he then obtained various results on the former ideals. Later, Eisenbud and Huneke showed that the Rees algebras of straightening-closed ideals are again ordinal Hodge algebras in a natural way $[E H]$. This enabled them to show that Rees algebras of certain determinantal ideals are Cohen-Macaulay.

Our viewpoint is to give a self-contained account of straightening-closed ideals, focusing largely on those properties that depend solely on the underlying Hodge algebra structure. As a main by-product, we recover most of the results of $[\mathrm{Hu} 2]$ and $[\mathrm{EH}]$. Moreover, some applications are given that do not

Received by the editors July 5, 1989.

1980 Mathematics Subject Classification (1985 Revision). Primary 13C05, 13C13, 13C15, $13 \mathrm{H} 10$.

Key words and phrases. Ordinal Hodge algebras, standard monomial, straightening-closed ideal, filtration of powers, Rees algebra, associated graded ring, normal, Cohen-Macaulay, Gorenstein, divisor class group, rank, arithmetical rank, generic matrix, virtual maximal minor.

A. Simis was partially supported by a CNPq grant No. 300662/82/MA. 
seem to have been explained from the Hodge algebra viewpoint. This unified treatment of the main examples, under the headline of straightening-closed ideals, has the effect of throwing clarity to the theory and conveying the usefulness of Hodge algebra methods in typical questions of commutative algebra.

\section{HODGE ALGEBRA APPROACH TO BLOW-UPS}

2.1. Straightening-closed ideals. Let $H$ be a finite set endowed with a partial order $\leq$ (briefly, a poset). A monomial on $H$ is a product $h_{1} \cdots h_{n}$ of elements of $H$. We say that $h_{1} \cdots h_{n}$ is standard if $h_{1} \leq \cdots \leq h_{n}$. Next, let $B$ be a commutative ring and $H$ a poset. A $B$-algebra $A$ is a graded ordinal Hodge algebra on $H$ over $B$ if the following conditions hold: $\left(\mathrm{H}_{0}\right) A=\bigoplus_{i \geq 0} A_{i}$ is a graded $B$-algebra with $B=A_{0}$, and $H \subset A$ is a set of elements of positive degree such that $A=B[H] ;\left(\mathrm{H}_{1}\right)$ the standard monomials on $H$ are linearly independent over $B ;\left(\mathrm{H}_{2}\right)$ for any two incomparable elements $h, k \in H$, there exists a relation $h k=\sum_{M} b_{M} M$, with $b_{M} \in B, b_{M} \neq 0$ and $M$ a standard monomial on $H$, for which every $M$ contains a factor $l \in H$ with $l \leq h$ and $l \leq k$.

We note that the relation required in $\left(\mathrm{H}_{2}\right)$ is uniquely determined by the pair $h, k$ because of $\left(\mathrm{H}_{1}\right)$-it is called the straightening of $h, k$. It follows that $A$ is a free $B$-module, the standard monomials serving as a basis (cf. [DEP, 1.1]); the representation of an element as a $B$-linear combination of standard monomials is called its standard representation. For the general aspects of the theory of Hodge algebras, the main reference will be the survey by DeConcini, Eisenbud and Procesi [DEP]. We are mainly interested in further structures derived from these algebras. As usual, an ideal in the poset $H$ is a subset $\Omega \subset H$ such that $k \in \Omega$ whenever $k \leq h \in \Omega$.

(2.1.1) Definition. Let $A$ be a graded ordinal Hodge algebra on a poset $H$. An ideal $\Omega \subset H$ is straightening-closed if, for every incomparable $h, k \in \Omega$, each standard monomial $M$ appearing in the straightening of $h, k$ contains at least two factors from $\Omega$.

The reader should check that the intersection of straightening-closed ideals is straightening-closed. We list some important examples of straightening-closed ideals:

(2.1.2) Example. Suppose that $A$ satisfies the following conditions (fulfilled by many important examples): (i) if $h, k \in H, h \leq k$, then $\operatorname{deg} h \geq \operatorname{deg} k$ (as homogeneous elements of $A$ ); (ii) each standard monomial in the straightening of a pair of incomparable elements contains at most two factors. Then the set $\Omega$ of elements of maximal degree in $H$ obviously is a straightening-closed ideal.

(2.1.3) Example (Ordinary maximal minors). Let $B$ be a commutative ring and $X$ a matrix of $m \times n$ indeterminates over $B$. Set $R=B[X]$. Then, as is well known, $A$ is a graded ordinal Hodge algebra over $B$ on the poset $\Delta(X)$ of all $r$-minors $\left[i_{1}, \ldots, i_{r} \mid j_{1}, \ldots, j_{r}\right]$ of $X, 1 \leq r \leq \min \{m, n\}$. Here, the partial 
order $\leq$ is defined by decreeing that

$$
\left[i_{1}, \ldots, i_{r} \mid j_{1}, \ldots, j_{r}\right] \leq\left[i_{1}^{\prime}, \ldots, i_{s}^{\prime} \mid j_{1}^{\prime}, \ldots, j_{s}^{\prime}\right]
$$

if and only if $r \geq s$ and $i_{1} \leq i_{1}^{\prime}, \ldots, i_{s} \leq i_{s}^{\prime}, j_{1} \leq j_{1}^{\prime}, \ldots, j_{s} \leq j_{s}^{\prime}$.

The straightening law on $B[X]$ has the following properties: (i) Every standard monomial on the right side of a straightening contains at most two factors. (ii) Every row index (column index) appears with the same multiplicity in all the monomials involved in a straightening.

Property (i) holds since the straightening relations in $B[X]$ can be viewed as $B$-linear combinations of dehomogenizations of the Plücker relations defining a Grassmannian in a suitable way (cf. [BV, Section 4]). This fact implies (ii), but (ii) also follows from the homogeneity of the minors with respect to the collection of indeterminates in a given row or column.

In conjunction with $(2.1 .2)$, property (i) implies immediately that the set $\Gamma(X)$ of the maximal minors of $X$ is a straightening-closed ideal.

(2.1.4) Example. (Ordinary maximal minors fixing a set of columns). Let $R$ be the Hodge algebra considered in the preceding example. For convenience, assume that $m \leq n$. Let $r$ be an integer such that $0 \leq r \leq m$ and let $\Gamma_{r}(X) \subset$ $\Gamma(X)$ denote the subset whose elements are the maximal minors involving the first $r$ columns of $X$. It follows immediately, from the properties (i) and (ii) of the straightening law in $B[X]$ as listed above, that $\Gamma_{r}(X)$ is a straighteningclosed ideal in $B[X]$.

(2.1.5) Example. (Virtual maximal minors in determinantal rings). First, we briefly describe what is meant here by determinantal rings-a complete account is to be found in [BV]. The shortest, although not the most explicit, way to introduce them is as follows. As in (2.1.3), let $\Delta(X)$ stand for the poset of all minors of $X$. Given $\delta \in \Delta(X)$, we set $\Delta(X ; \delta)=\{\pi \in \Delta(X) \mid \pi \geq \delta\}$. The complementary set $\Omega(X ; \delta)=\Delta(X) \backslash \Delta(X ; \delta)$ is clearly an ideal in the poset $\Delta(X)$. Denote by $I(X ; \delta)$ the ideal of the ring $B[X]$ generated by $\Omega(X ; \delta)$ and let $R(X ; \delta)=B[X] / I(X ; \delta)$.

It follows from the general theory that $R(X ; \delta)$ is again a graded ordinal Hodge algebra over $B$ on the poset $\Delta(X ; \delta)$, called the determinantal ring of type $(X ; \delta)$. A special notable case is the determinantal ring of type $(X ;[1 \cdots t-1 \mid 1 \cdots t-1])$ since it coincides with the residue class ring of $B[X]$ modulo the ideal $I_{t}(X)$ generated by the $t$-minors of $X$.

Continuing, let $\delta=\left[a_{1} \cdots a_{r} \mid b_{1} \cdots b_{r}\right]$. For given integers $1 \leq k \leq r$ and $a_{k}<\tilde{a}_{k} \leq a_{k+1}$, we let $\varepsilon_{k}^{a_{k}} \in \Delta(X ; \delta)$ be the smallest element such that $\varepsilon_{k}^{\tilde{a}_{k}}=\left[a_{1} \cdots a_{k-1} \tilde{a}_{k} \cdots \tilde{a}_{\tilde{r}} \mid b_{1} \cdots b_{\tilde{r}}\right]$ (clearly, $\left.\tilde{r} \leq r\right)$. One can see that the ideals $I\left(X ; \varepsilon_{k}^{a_{k}}\right)$ and $I(X ; \delta)$ contain the same minors up to order $t \leq k-1$. Moreover, the condition $\tilde{a}_{k} \leq a_{k+1}$ ensures that the $(k+1)$-minors in $I\left(X ; \varepsilon_{k}^{\tilde{a}_{k}}\right)$ already belong to $I(X ; \delta)$. Therefore, the ideal $I\left(X ; \varepsilon_{k}^{\tilde{a}_{k}}\right) / I(X ; \delta)$ of the determinantal ring $R(X ; \delta)$ is generated by the $k$-minors of the rows $1, \ldots, \tilde{a}_{k}-1$ 
of $X$-we call it an ideal of virtual maximal minors of $R(X ; \delta)$. A similar construction is possible with the columns of $X$. Notable instances are:

(1) $\delta=[1, \ldots, r \mid 1, \ldots, r], \varepsilon_{r}^{m+1}=[1, \ldots, r-1 \mid 1, \ldots, r-1]$. Here, $I\left(X ; \varepsilon_{r}^{m+1}\right) / I(X ; \delta)=I_{r}(X) / I_{r+1}(X) \subset B[X] / I_{r+1}(X)$.

(2) $\delta=[1, \ldots, r \mid 1, \ldots, r], \varepsilon_{r}^{r+1}=[1, \ldots, r-1, r+1 \mid 1, \ldots, r]$. Here,

$$
I\left(X ; \varepsilon_{r}^{r+1}\right) / I(X ; \delta)=I_{r}\left(X_{\mid r}\right) / I_{r+1}(X) \subset B[X] / I_{r+1}(X),
$$

where $X_{\mid r}$ stands for the $r \times n$ submatrix of $X$ formed by the first $r$ rows.

$\Omega(X ; \delta ; \varepsilon)=\Delta(X ; \delta) \cap \Omega(X ; \varepsilon)$ is an ideal in $\Delta(X ; \varepsilon)$. We claim that, for $\varepsilon=\varepsilon_{k}^{a_{k}}$ as above, the ideal $\Omega(X ; \delta ; \varepsilon)$ is straightening-closed. We sketch the proof of this fact. Let $\xi, v \in \Omega(X ; \delta ; \varepsilon)$ be incomparable elements, and let $\xi v=\sum b_{M} M, b_{M} \neq 0$, be the corresponding straightening, where, typically, $M=\mu_{1} \mu_{2}$, with $\mu_{1} \leq \mu_{2} \in \Delta(X ; \delta)$ and $\mu_{1} \leq \xi, v$. Now, an element of $\Delta(X ; \delta)$ belonging to $\Omega(X ; \delta ; \varepsilon)$ is characterizable by having exactly $k$ row indices strictly less than $\tilde{a}_{k}$. Since the union (with multiplicities) of the row indices is constant along the monomials, we must have $\mu_{2} \in \Omega(X ; \delta ; \varepsilon)$ as well.

(2.1.6) Example. (Virtual maximal minors in Schubert cycles). This example is similar to the preceding one, therefore we skip the details (cf. [BV]). Let $G(X) \subset B[X]$ be the $B$-subalgebra generated by the set $\Gamma(X)$ of maximal minor of the $m \times n$ generic matrix $X$. As is well-known, $G(X)$ is a graded ordinal Hodge algebra on $\Gamma(X)$ over $B$. Given $\delta \in \Gamma(X)$, we let $\Gamma(X ; \delta)=\{\pi \in \Gamma(X) \mid \pi \geq \delta\}, J(X ; \delta)=G(X)(\Gamma(X) \backslash \Gamma(X ; \delta))$ and $G(X ; \delta)=G(X) / J(X ; \delta)$. The algebras $G(X ; \delta)$ arise as the homogeneous coordinate rings of the Schubert subvarieties of the Grassmannian. Note that, as in the preceding example, $G(X ; \delta)$ is a graded ordinal Hodge algebra on $\Gamma(X ; \delta)$ over $B$. Virtual maximal minors in $G(X ; \delta)$ are defined in entire analogy with the preceding example.

(2.1.7) Example. (Union of two skew linear subspaces). Let $B$ be a commutative ring, $X_{1}, \ldots, X_{n}$ and $Y_{1}, \ldots, Y_{m}$ two independent sets of indeterminates over $B$, and $R=B[X, Y]$. One endows the set $H=\left\{X_{i}, Y_{j}, X_{i} Y_{j} \mid 1 \leq i \leq n, 1 \leq\right.$ $j \leq m\}$ with a poset structure as follows:

$$
\begin{gathered}
X_{1} \leq \cdots \leq X_{n}, \quad Y_{1} \leq \cdots \leq Y_{m}, \quad X_{i_{1}} Y_{j}<X_{i_{2}} \Leftrightarrow i_{1} \leq i_{2}, \\
X_{i} Y_{j_{1}}<Y_{j_{2}} \Leftrightarrow j_{1} \leq j_{2}, \quad X_{i_{1}} Y_{j_{1}} \leq X_{i_{2}} Y_{j_{2}} \Leftrightarrow i_{1} \leq i_{2} \quad \text { and } \quad j_{1} \leq j_{2} .
\end{gathered}
$$

One can think of $H$ as the poset of entries of a suitable matrix with the greatest element deleted. It is an easy exercise to see that $R$ is indeed a graded ordinal Hodge algebra on $H$ over $B$.

Let $\Omega=\left\{X_{i} Y_{j} \mid 1 \leq i \leq n, 1 \leq j \leq m\right\}$. Clearly, $\Omega$ is a straightening-closed ideal in $H$. Note that $\Omega R=(X) R \cap(Y) R$, justifying the terminology "union of two skew spaces". This ideal may be useful in studying the 2-minors of a generic matrix (cf. (4.3)). 
2.2. Graded algebras derived from a straightening-closed ideal. We recall the following notions. Let $I \subset R$ be an ideal. The Rees algebra of $I$ is the graded $R$-algebra $\mathscr{R}(I)=R \oplus I \oplus I^{2} \oplus \cdots$. If $I$ is generated by a set $\left\{a_{1}, \ldots, a_{m}\right\}$, then $\mathscr{R}(I)$ admits a presentation $\mathscr{R}(I) \simeq R[T] / J$, where $T=T_{1}, \ldots, T_{m}$ are indeterminates over $R$ and one assigns $T_{i} \rightarrow a_{i}, 1 \leq i \leq m$. By a similar token, the symmetric algebra $\mathscr{S}(I)$ of $I$ admits the $R$-algebra presentation $\mathscr{S}(I) \simeq R[T] / J_{1}$ where $J_{1} \subset J$ is the ideal generated by the 1 -forms of $J$. Thus, we have a surjection $\mathscr{S}(I) \rightarrow \mathscr{R}(I)$, which is an isomorphism if and only if $J$ is generated by its 1 -forms.

Observe that $\mathscr{R}(I)$ can be embedded in the polynomial ring $R[t]$ as the $R$-subalgebra $R[I t]$. If, moreover, $R$ has a structure of graded ordinal Hodge algebra on a poset $H \subset R$ over a ring $B$, and if $I$ is generated by the elements of a straightening-closed ideal $\Omega \subset H$, then $R[I t]$ can be endowed with a structure of graded ordinal Hodge algebra over $B$ on a suitable poset. This procedure was discussed by Eisenbud and Huneke [EH]. The underlying poset is $H \cup \Omega t \subset R[I t]$ (the elements of $H$ being regarded as degree zero elements of $R[I t])$, partially ordered by decreeing: $H$ is to have its original ordering, $\Omega t$ is to be ordered via the identification $\delta \leftrightarrow \delta t, \delta \in \Omega$, and if $h \in H, \delta t \in \Omega t$, then $\delta t<h$ if and only if $\delta<h$ in $H$.

In this way, $R[I t]$ is a graded ordinal Hodge algebra on $H \cup \Omega t$ in a natural way. We refer to $[\mathrm{EH}]$ for the details and content ourselves with the description of the straightening relations. There are four types, the first three derived from a straightening $h k=\sum_{M} b_{M} M$ of incomparable elements $h, k \in H$, and the last one representing the trivial relation $h k=k h$ :

$$
\begin{aligned}
k h & =\sum_{M} b_{M} M, \quad h, k \in H, \\
h t k & =\sum_{M} b_{M}\left(x_{M} t\right) y_{M} N_{M}, \quad h \in \Omega, k \in H, \\
h t k t & =\sum_{M} b_{M}\left(x_{M} t\right)\left(y_{M} t\right) N_{M}, \quad h, k \in \Omega, \\
h t k & =k t h, \quad h, k \in \Omega, h>k,
\end{aligned}
$$

where $M=x_{M} y_{M} N_{M}$, with $x_{M} \leq y_{M} \leq z, z \in H$ a factor of $N_{M}$. (Note that the assumption on $\Omega$ being straightening-closed warrants that, besides $x_{M}$, also $y_{M} \in \Omega$.)

Now, using a presentation $R[I t] \simeq R[T] / J$ to read the straightening relations as homogeneous polynomials in $T$, one easily obtains

(2.2.1) Lemma. The presentation ideal $J$ of $R[I t]$ is generated by the 1-forms $k T_{h}-h T_{k}, h>k$, and $k T_{h}-\sum_{M} b_{M} y_{M} N_{M} T_{x_{M}}, k \in H, h \in \Omega$, and the 2-forms $T_{h} T_{k}-\sum_{M} b_{M} N_{M} T_{x_{M}} T_{y_{M}}, h, k \in \Omega$.

Proof. Combine [DEP, Proposition 1.1] with the above description of the straightening relations in $R[I t]$. 
Of course, the representation of $R[I t]$ is given in (2.2.1) is not minimal in general, neither with respect to the generating set $\Omega t$, nor with respect to the relations. We now state the main result of this section:

(2.2.2) Theorem. Let $R$ be a graded ordinal Hodge algebra on a poset $H$ over a ring $B$, and $\Omega \subset H$ a straightening-closed ideal.

(a) Suppose that $\Omega$ is linearly ordered.

(i) Then the elements of $\Omega$ forms a d-sequence.

(ii) In particular the symmetric and the Rees algebra of $\Omega R$ coincide.

(b) Suppose that every standard monomial in a straightening relation $h k=$ $\sum a_{M} M, h \in H, k \in \Omega$, contains at least two factors. Then (ii) implies that $\Omega$ is linearly ordered.

Proof. (a) The notion of $d$-sequence was introduced by Huneke and the implication (i) $\Rightarrow$ (ii) is the main result of [Hu 1]; of course, (ii) follows also directly from (2.2.1): the representation ideal $J$ of $\mathscr{R}(I), I=\Omega R$, is generated by linear forms since there are no straightening relations of the third type.

In order to show that the elements of $\Omega=\left\{h_{1}, \ldots, h_{n}\right\}, h_{1}<\cdots<h_{n}$, form a $d$-sequence we may argue inductively modulo a subideal $\left\{h_{1}, \ldots, h_{m}\right\}$. Therefore it is enough to show: if $a h_{j} \neq 0, a \in R, j \geq 1$, then $a h_{1} h_{j} \neq 0$. Consider the standard representation $a h_{j}=\sum b_{M} M, M$ a standard monomial. Since every $M$ has to start with a factor $\leq h_{j}$, hence with one among $h_{1}, \ldots, h_{j}, h_{1} M$ is again a standard monomial, and $a h_{j} h_{1}=\sum b_{m} h_{1} M$ is the standard representation of $a h_{j} h_{1}$.

(b) The hypothesis implies that the coefficients of the 1-forms, among the generators of the representation ideal $J$ of $\mathscr{R}(I)$ are contained in $H R$. If there are incomparable elements $h, k \in \Omega$, then there is a 2 -form with a unit coefficient in $J$.

Since $J$ is generated by its forms of degree $\leq 2$, the equality of $\mathscr{S}(I)$ and $\mathscr{R}(I)$ can be replaced by the weaker equation $\mathscr{S}^{2}(I)=I^{2}$. Ideals with the latter property were called syzygetic in [SV], and they are precisely the ideals for which the deformation functor $T_{2}^{S / R}, S=R / I$, vanishes.

Let $R$ be a graded ordinal Hodge algebra on a poset $H$ and let $\Omega \subset R$ be an ideal. Set $I=\Omega R$. In general $\operatorname{gr}_{I}(R)$ is not an ordinal Hodge algebra as the following simple example indicates: $H=\Delta(X)$, with $X$ a generic $3 \times 3$ matrix and $\Omega \subset H$ the ideal consisting of the 2-minors and the determinant $\delta$ of $X$. Letting $*$ denote leading form in $\operatorname{gr}_{I}(R)$, one has $\delta \notin I^{2}$, so $\delta^{*} \in$ $I / I^{2} \subset \operatorname{gr}_{I}(R)$. On the other hand, a typical straightening is [12|13][13|12] = [12|12][13|13] $-\delta X_{11}$, which, incidentally, shows that $\Omega$ is not straighteningclosed. By manipulating this relation and its conjugates, one sees that $\delta^{2} \in I^{3}$, so $\left(\delta^{*}\right)^{2}=0$. For straightening-closed ideals the situation is much better. 
(2.2.3) Lemma. Let $R$ be a graded ordinal Hodge algebra over $B$ on a poset $H$ and let $\Omega \subset H$ be a straightening-closed ideal. For $I=\Omega R$, the filtration $\left\{I^{n}\right\}$ is a standard filtration (in the sense of [DEP, §2]).

Proof. It suffices to show that, for every $n \geq 0, I^{n}$ is the $B$-submodule of $R$ generated by all standard monomials in $H$ with at least $n$ factors from $\Omega$. Thus one has to show that every standard monomial appearing in the standard representation of a monomial containing at least $n$ factors from $\Omega$, contains at least $n$ factors from $\Omega$, too. For this one introduces a sort of coheight function: For an element $h \in H$, set $v(h)=\#\{g \in H \mid g>h\}$. By a descending induction, using the measure $2^{v(h)}$, by the fact that a standard representation of a monomial is obtained by successively applying the straightening to two incomparable elements, and by the defining property of a straightening-closed ideal, one is through with the claim.

(2.2.4) Proposition. Let $R$ be a graded ordinal Hodge algebra, and let $\Omega$ be a straightening-closed ideal in the defining poset $H$ of $R$. If $I=\Omega R$ then $\operatorname{gr}_{I}(R)$ is a graded ordinal Hodge algebra on the poset $H^{*}$ of the corresponding leading forms.

The proposition is a well-known consequence of the lemma ([DEP]; cf. also [BV, (9.8)]).

(2.2.5) Corollary. Let $R$ be a graded ordinal Hodge algebra over $B$ on a poset $H$. Let $\Omega \subset H$ be a straightening-closed ideal and $I=\Omega R$.

(i) If $B$ is reduced, then $\operatorname{gr}_{I}(R)$ is reduced.

(ii) If $B$ is Cohen-Macaulay and if $H$ is wonderful, then $\operatorname{gr}_{I}(R)$ is CohenMacaulay.

Proof. (i) is well known [DEP]. As for (ii), again by [DEP], it suffices to remark that $H$ and $H^{*}$ are isomorphic posets.

2.3. Filtrations of powers of straightening-closed ideals. In this section we introduce filtrations for the powers of $I$, where $I$ is an ideal of a graded ordinal Hodge algebra $R$ generated by a straightening-closed ideal in the defining poset $H$ of $R$. Similar filtrations were considered by Huneke [Hu 2].

For $h \in H$ set $w(h)=2^{v(h)}$, where $v(h)$ is defined as in the proof of (2.2.3). For a monomial $M=h_{1} \cdots h_{p}$ we further set $w(M)=\sum w\left(h_{i}\right)$. More generally, for any integer $n \geq 0$, introduce a function $w_{n}(M)=\sum_{i=1}^{\min (n, p)} w\left(h_{i}\right)$, where we assume that $w\left(h_{1}\right) \geq \cdots \geq w\left(h_{p}\right)$.

(2.3.1) Lemma. Let $R$ be a graded ordinal Hodge algebra and let $M=\sum b_{i} M_{i}$ be a standard representation of the monomial $M$. Then $w_{n}\left(M_{i}\right) \geq w_{n}(M)$ for all $i, n$.

Proof. We proceed by descending induction on $w(M)$. If $M$ is standard, there is nothing to prove. Otherwise, $M=h_{1} \cdots h_{p}$ with, say, $h_{i}$ and $h_{j}$ incomparable $(i<j)$. Let $h_{i} h_{j}=\sum b_{k} N_{k}$ be their straightening. Then

$$
M=\sum b_{k}\left(h_{1} \cdots \hat{h}_{i} \cdots \hat{h}_{j} \cdots h_{p}\right) N_{k}
$$


and one clearly has $w\left(\left(h_{1} \cdots \hat{h}_{i} \cdots \hat{h}_{j} \cdots h_{p}\right) N_{k}\right)>w(M)$. Applying descending induction, it remains to verify

$$
w_{n}\left(\left(h_{1} \cdots \hat{h}_{i} \cdots \hat{h}_{j} \cdots h_{p}\right) N_{k}\right) \geq w_{n}(M),
$$

which can be done by analysing the cases where $i, j \leq n, i \leq n<j$, and $n \leq i, j$, respectively.

Let, as before, $R$ be a graded ordinal Hodge algebra on a poset $H$. The length of a monomial in $H$ is the number of its factors from $H$. Given a straightening-closed ideal $\Omega \subset H$, one sets

$$
\Omega^{(n)}=\{M \mid M \text { a standard monomial of length } n \text { in } \Omega\} .
$$

The set $\Omega^{(n)}$ can be partially ordered by decreeing:

$$
M \leq N \Leftrightarrow M=N \text { or } w(M)<w(N) .
$$

Further, for a standard monomial $M=h_{1} \cdots h_{p}, h_{1} \leq \cdots \leq h_{p}$, one puts $\alpha(M)=h_{1}, \omega(M)=h_{p}$ and, more generally, $\alpha_{i}(M)=h_{1} \cdots h_{\min (i, p)}, \omega_{i}(M)=$ product of the remaining factors of $M$ (so that $M=\alpha_{i}(M) \omega_{i}(M)$ ). The tail coideal associated to the standard monomial $M$ is

$$
\Psi(M)=\{h \in H \mid h<\omega(M) \text { or } h, \omega(M) \text { are incomparable }\} .
$$

(2.3.2) Proposition. For every $n \geq 1$, the ideal $I^{n}(I=\Omega R)$ has a finite filtration by ideals whose quotients are cyclic $R$-modules of the form $R / \Psi(h) R$, $h \in \Omega$.

Proof. One defines the general term of the filtration as follows:

$$
J_{j}=\bigoplus_{M} B M,
$$

where $M$ runs through the standard monomials such that $\alpha_{n}(M) \in \Omega^{(n)}$ and $w_{n}(M) \geq j$. First one observes that if $M$ is an arbitrary monomial in $H$ such that $M \in I^{n}$ and $w_{n}(M) \geq j$, then $M \in J_{j}$-this follows from (2.2.3) (or rather from its proof) and from (2.3.1). Next, $J_{j}$ is indeed an ideal of $R$. For this it suffices to show that $H M \in J_{j}$ for any standard monomial $M \in J_{j}$. Let $h \in H$. By the earlier observation it is enough to have $h M \in I^{n}$ and $w_{n}(h M) \geq j$. But the first is clear, while $w_{n}(h M) \geq w_{n}(M) \geq j$.

We next show: if $M \in \Omega^{(n)}$ and $w_{n}(M)=j$ then $\Psi(M) M \subset J_{j+1}$. Let $h \in \Psi(M)$. Every standard monomial in the standard representation $h M=$ $\sum b_{N} N$ satisfies $\alpha_{n}(N) \in \Omega^{(n)}$ by the proof of (2.2.3). It remains to show that $w_{n}(N) \geq j+1$ for all these standard monomials. Assume first that $h<\omega(M)$. Then $w_{n}(h M)=w_{n-1}(M)+w(h)>w_{n-1}(M)+w(\omega(M))=j$, and one is through by (2.3.1). Otherwise $h$ and $\omega(M)$ are not comparable; let $h \omega(M)=$ $\sum b_{i} N_{i}$ be the standard representation. Then

$$
h M=\sum b_{i} \alpha_{n-1}(M) \alpha\left(N_{i}\right) \omega_{1}\left(N_{i}\right)
$$

and $\alpha\left(N_{i}\right)<\omega(M)$, in particular $w_{n}\left(\alpha_{n-1}(M) \alpha\left(N_{i}\right) \omega_{1}\left(N_{i}\right)\right)>w_{n}(M)$ as above. Now one applies (2.3.1) again. 
Finally, we claim that there is an isomorphism of $R$-modules

$$
J_{j} / J_{j+1} \cong \bigoplus_{\substack{M \in \Omega^{(n)} \\ w_{n}(M)=j}} R / \Psi(M) R
$$

Take the free $R$-module on the basis $\left\{e_{M}\right\}, M \in \Omega^{(n)}, w_{n}(M)=j$, and define a surjective map $\pi: F \rightarrow J_{j} / J_{j+1}$ by $e_{M} \rightarrow M$. The preceding claim shows that $\bigoplus R \Psi(M) e_{M} \subset \operatorname{Ker}(\pi)$. Moreover, a comparison of bases yields that, as $B$ modules, $J_{j}=J_{j+1} \oplus K$, where $K$ is generated by the standard monomials $N=\alpha_{n}(N) \omega_{n}(N)$ such that $\alpha_{n}(N) \in \Omega^{(n)}$ and $w_{n}\left(\alpha_{n}(N)\right)=j$. Necessarily, $\alpha\left(\omega_{n}(N)\right) \geq\left(\omega\left(\alpha_{n}(N)\right)\right.$ if $\omega_{n}(N) \neq 1$. Thus, for fixed $M=\alpha_{n}(N)$, the tails $\omega_{n}(N)$ are exactly the standard monomials which constitute the standard basis of $R / \Psi(M) R$.

As a consequence, the modules $I^{m} / I^{n}$ admit similar filtrations:

(2.3.3) Corollary. For every $n>m$, the module $I^{m} / I^{n}$ has a finite filtration whose quotients are cyclic modules of the form $R /(\Omega \cup \Psi(h)) R, h \in \Omega$ or, if $m=0, R / I$.

Proof. It is clearly enough to display such a filtration for the $n$th conormal module $I^{n} / I^{n+1} \quad(n \geq 1)$. Consider the filtration $\left(J_{j}\right)$ constructed in the proof of (2.3.2) and set $\bar{J}_{j}=\left(J_{j}+I^{n+1}\right) / I^{n+1}$. For the induced surjection $\bar{\pi}: F \rightarrow \bar{J}_{j}$ one has $\bigoplus_{M}(\Omega \cup \Psi(M)) \operatorname{Re}_{M} \subset \operatorname{Ker}(\bar{\pi})$. Comparing standard bases yields equality.

(2.3.4) Remark. Under the assumption of (2.3.2), suppose that $B$ is noetherian and let $S=R / I$. We claim: for every $P \in$ Ass $S$, the $S_{P}$-module $\left(I^{n} / I^{n+1}\right)_{P}$ is free. $S$ is a graded ordinal Hodge algebra over $B$. This implies that $S \otimes$ $B / Q, Q=B \cap P$ is reduced, and together with the flatness of the extension $B \rightarrow S$ one easily obtains $Q S_{P}=P S_{P}$. Note that $I^{n} / I^{n+1}$ is a free $B$-module by the proof of (2.3.3). Setting $T_{1}=B_{Q}, T_{2}=S_{P}, M=\left(I^{n} / I^{n+1}\right)_{P}$, we are left with the following situation: $T_{1} \rightarrow T_{2}$ is a flat local homomorphism such that $\mathbf{m}_{1} T_{2}=\mathbf{m}_{2}$ for the maximal ideals $\mathbf{m}_{1}$ and $\mathbf{m}_{2}$ respectively, and the finitely generated $T_{2}$-module $M$ is flat over $T_{1}$. Then it is easy to see that $M$ is flat over $T_{2}$, hence a free $T_{2}$-module (cf. [Ma, (20.G)]).

\section{MAIN THEOREMS}

3.1. Powers of straightening-closed ideals are primary. We define the symbolic powers of an ideal $I \subset R$ by $I^{(n)}=\left\{x \in R \mid y x \in I^{n}\right.$ for some non-zero-divisor $y$ on $R / I\}$. The symbolic Rees algebra of $I$ is the graded $R$-algebra

$$
\mathscr{R}^{(I)}=R \oplus I \oplus I^{(2)} \oplus I^{(3)} \oplus \cdots .
$$

A question that has called the attention of several algebraists in recent years, is to find sufficient conditions for the equality $\mathscr{R}(I)=\mathscr{R}^{(I)}$. The following 
simple examples may indicate that this is a hard problem, even in the context of graded ordinal Hodge algebras, since not just the nature of the poset, but also that of the straightening relations plays a role. Let $k$ be a field, $R=k[x, y, z]$ a graded ordinal Hodge algebra on the poset $\{x, y, z\}$, partially ordered by $z<x, z<y$, and $\Omega=\{x, z\}$. If $x y=0$ (the discrete case), then $x \in$ $I^{(2)} \backslash I^{2}$. Similarly, if $x y=z^{2}$, then $x \in I^{(2)} \backslash I^{2}$. If, however, $x y=z$, then $\mathscr{R}(I)=\mathscr{R}^{(I)}$.

The best possible general result seems to be following theorem. In a slightly different set-up it goes back at least to [Hu 2] and is based on the tail coideal filtrations of (2.3).

(3.1.1) Theorem. Let $B$ be a noetherian ring and $R$ a graded ordinal Hodge algebra on a poset $H$. Let $\Omega \subset H$ be a straightening-closed ideal and $I=\Omega R$. The following conditions are equivalent:

(i) $I^{n}=I^{(n)}$ for every $n \geq 0$.

(ii) $I_{P}^{n}=\left(I_{P}\right)^{(n)}$ for every $n \geq 0$ and for every associated prime ideal $P$ of any of the rings $R /(\Omega \cup \Psi(h)) R, h \in \Omega$.

Proof. (i) $\Rightarrow$ (ii) We must prove the torsionfreeness of $\left(I^{n} / I^{n+1}\right)_{Q}$ over $(R / I)_{Q}, Q=P / I$, for at least the prime ideals $P$ named in (ii). Let $M=$ $I^{n} / I^{n+1}$. Since $M_{Q}$ is a free $S_{Q}$-module for $Q \in$ Ass $S$ by (2.3.4), the torsion of $M$ coincides with the kernel of the natural homomorphism of $M$ into its $S$-bidual, and the formation of the latter commutes with localization.

(ii) $\Rightarrow$ (i) One has to show that if a prime ideal $P \subset R$ is associated to $R / I^{n}$ for some $n$, then $P$ is also associated to $R / I$. At any rate, $I \subset P$. From the exact sequence

$$
0 \rightarrow I / I^{n} \rightarrow R / I^{n} \rightarrow R / I \rightarrow 0
$$

and (2.3.3), one has

$$
\text { Ass } R / I^{n} \subset(\text { Ass } R / I) \cup \bigcup_{h \in \Omega} \text { Ass } R /(\Omega \cup \Psi(h)) R .
$$

Under "regularity" assumptions it has recently been proved that the equality $\mathscr{R}(I)=\mathscr{R}^{(I)}$ takes place if $\operatorname{gr}_{I}(R)$ is reduced [HuSV]. Thus, straighteningclosed ideals provide a natural environment under which the above equality holds, since their associated graded rings are reduced over reduced rings of coefficients, cf. (2.2.5).

(3.1.2) Theorem. Let $B$ be a reduced noetherian ring, let $R$ be a graded ordinal Hodge algebra over $B$ on a poset $H$ and let $\Omega \subset H$ be a straightening-closed ideal. Set $I=\Omega R$. Suppose that the following conditions hold:

(i) $R / I$ has finite homological dimension over $R$.

(ii) $R$ is locally formally equidimensional.

Then $I^{n}=I^{(n)}$ for every $n \geq 0$. 
As we will see below, one can first pass from $B$ to the residue class fields $(B / p)_{p}, p$ a prime ideal in $B$, before applying (3.1.2); thus, one may essentially assume that $B$ is a field. Then $B$ is reduced, condition (ii) usually holds, and (i) is satisfied if $R$ is a polynomial ring over $B$.

3.2. Arithmetic of blowups of straightening-closed ideals. One of the general questions posed in [ST] is the computation of the divisor class group of a normal Rees algebra. The results obtained there can be applied in the present situation.

(3.2.1) Theorem. Let $B$ be a normal domain and let $R, H, \Omega, I$ satisfy the hypotheses of (3.1.2). Then the Rees algebra $\mathscr{R}(I)$ is normal and $\mathrm{Cl}(\mathscr{R}(I)) \simeq$ $\mathrm{Cl}(R) \oplus \mathbf{Z}^{r}$, where $r$ is the number of associated primes of $R / I$ of height at least two.

Proof. Since $\operatorname{gr}_{I}(R)$ is reduced and $R$ is normal, $\mathscr{R}(I)$ is normal. Since $R / I$ is reduced and of finite homological dimension, the localizations $R_{P}$, $P \in$ Ass $R / I$, are regular. On the other hand, by (3.1.2), $\operatorname{gr}_{I}(R)$ is torsionfree over $R / I$. In this case, the required expression for $\mathrm{Cl}(\mathscr{R}(I))$ has been given in [ST].

In the absence of a condition of regularity, such as conditions (i) and (ii) of (3.1.2), one still has, quite generally, an exact sequence of divisor class groups

$$
0 \rightarrow F \rightarrow \mathrm{Cl}(\mathscr{R}(I)) \rightarrow \mathrm{Cl}(R) \rightarrow 0,
$$

where $F$ is freely generated by the classes of the height one primes containing $I \mathscr{R}(I)$ whose contraction to $R$ have height at least two [ST]. The questions of computing the rank of $F$ and of determining general conditions under which the above sequence splits has been treated in [HSV 2]. We next provide a different approach for the splitting question in the context of Hodge algebras.

Recall Samuel's condition (PDE) [Fo, p. 30] for an inclusion of normal domains $B \subset A$ : if $P \subset A$ is a height one prime then the height of $P \cap B$ is at most one. We introduce one further notion: the extension $B \subset A$ is unramified in codimension one if, for every height one prime $p \subset B$, either $p A_{p}$ is a prime ideal of $A_{p}$ or else $p A_{p}=A_{p}$.

The following lemma may be found in the standard literature, although we lack an adequate reference.

(3.2.2) Lemma. Let $B \subset A$ be an extension of normal domains which is unramified in codimension one and satisfies the property (PDE). Then there is a natural exact sequence

$$
\mathrm{Cl}(B) \stackrel{\iota}{\longrightarrow} \mathrm{Cl}(A) \stackrel{\kappa}{\longrightarrow} \mathrm{Cl}\left(A_{B \backslash 0}\right) \rightarrow 0 .
$$

If $A$ is a graded $B$-algebra such that $B=A_{0}$, then the map $\mathrm{Cl}(B) \rightarrow \mathrm{Cl}(A)$ is injective.

Proof. As a consequence of (PDE) there is a natural map $\mathrm{Cl}(B) \stackrel{l}{\rightarrow} \mathrm{Cl}(A)$ defined by

$$
l\left(\mathrm{cl}_{B}(p)\right)=\sum_{P \cap B=p} v(P / p) \mathrm{cl}_{A}(P),
$$


where $p A_{P}=P_{P}^{v(P / p)}$. The ramification condition implies that either $l\left(\operatorname{cl}_{B}(p)\right)$ $=0$, or else there is a single prime $P \subset A$ lying over $p$ and $v(P / p)=1$, in which case $l\left(\mathrm{cl}_{B}(p)\right)=\mathrm{cl}_{A}(P)$. Now, let $\kappa$ be induced by taking fractions. Then $\operatorname{Ker} \kappa$ is generated by $\left\{\mathrm{cl}_{A}(P) \mid P \cap B \neq 0\right\}$ and, from what we have just seen, it coincides with $\operatorname{Im} l$. The second statement is [Fo, Proposition 10.7(c)].

(3.2.3) Corollary. Let $R$ be a graded ordinal Hodge algebra over $B$ on a poset $H$, and let $I \subset R$ be an ideal generated by a straightening-closed ideal in $H$. Assume:

(i) $R$ is a normal domain and the extension $B \subset R$ is unramified in codimension one.

(ii) $\mathrm{Cl}\left(R_{B \backslash 0}\right)$ is a free $\mathrm{Z}$-module.

Then $\mathscr{R}(I)$ is normal and $\mathrm{Cl}(\mathscr{R}(I)) \simeq \mathrm{Cl}(B) \oplus \mathbf{Z}^{r+s}$, where $r$ is the number of minimal primes of $\mathrm{gr}_{I}(R)$ whose contraction to $R$ have height at least two and $s=\operatorname{rank} \mathrm{Cl}\left(R_{B \backslash 0}\right)$.

Proof. We first apply [ST, 3.1]. One has a natural commutative diagram

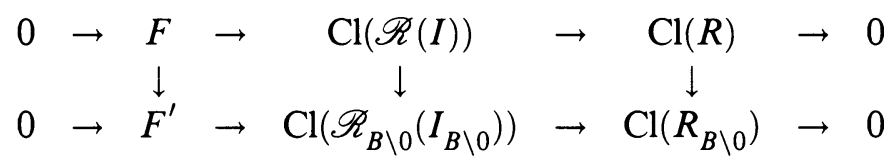

whose bottom row splits by hypothesis. Observe that, for every prime ideal $P \subset \mathscr{R}(I), P \in \operatorname{Ass}\left(\operatorname{gr}_{I}(R)\right)$, the intersection $P \cap B$ is 0 , since $\operatorname{gr}_{I}(R)$ is a free $B$-module. Therefore these prime ideals correspond bijectively to the analogous ones in $\mathscr{R}_{B \backslash 0}\left(I_{B \backslash 0}\right)$. Hence the map $F \rightarrow F^{\prime}$ is an isomorphism, and one obtains the splitting $\mathrm{Cl}(\mathscr{R}(I)) \simeq \mathrm{Cl}(R) \oplus F$.

The extension $B \subset R$ satisfies (PDE) since $R$ is a free $B$-module. Now (3.2.2) yields that $\mathrm{Cl}(R) \simeq \mathrm{Cl}(B) \oplus \mathrm{Cl}\left(R_{B \backslash 0}\right)$.

We will observe in $\S 4$ that the hypotheses of (3.2.3) are satisfied in many important cases. Nevertheless it would be interesting to find more general conditions under which $\mathrm{Cl}(R)$ splits off $\mathrm{Cl}(\mathscr{R}(I))$.

The question as to when the associated graded ring $\operatorname{gr}_{I}(R)$ is Gorenstein has been considered by various authors. A specialization of [HSV 2, 4.2.2] to the present situation yields the following result:

(3.2.4) Proposition. Let $B$ be a Cohen-Macaulay normal domain and a residue class ring of a Gorenstein ring, and let $R, H, \Omega, I$ satisfy the hypotheses of (3.1.2). Suppose moreover that $H$ is a wonderful poset and $\Omega$ is a self-covering ideal containing all the minimal elements of $H$. Then the following conditions are equivalent:

(i) $\operatorname{gr}_{I}(R)$ is Gorenstein.

(ii) $R$ is Gorenstein and $I$ is unmixed. 
Proof. By (3.1.2), $\operatorname{gr}_{I}(R)$ is torsionfree over $R / I$, and, by $(3.2 .1), \mathscr{R}(I)$ is normal. The hypothesis on $H$ and $\Omega$ implies that $\mathscr{R}(I)$ is Cohen-Macaulay, cf. [BV, (9.12)]. Now [HSV 2, 4.2.2] can be applied.

3.3. Depth bounds for powers of straightening-closed ideals. As before, let $R$ denote a graded ordinal Hodge algebra over a noetherian ring $B$ on a poset $H$. For a graded $R$-module $M$, depth $M$ will denote the length of a maximal $M$-sequence in $H R$.

(3.3.1) Proposition. Let $\Omega \subset H$ be a straightening-closed ideal and $I=\Omega R$. Then:

(i) $\operatorname{depth} I^{n} \geq \min _{h \in \Omega} \operatorname{depth} R / \Psi(h) R$.

(ii) $\operatorname{depth} R / I^{n} \geq \min \left\{\operatorname{depth} R, \min _{h \in \Omega}\{(\operatorname{depth} R / \Psi(h) R-1)\}\right\}$.

The depth bound in (ii) holds for $I^{n} / I^{n+1}$ as well.

Proof. (i) is a direct consequence of (2.3.2). (ii) follows from (i) in the obvious way. Since $\min _{n} \operatorname{depth} I^{n} / I^{n+1}=\min _{n} \operatorname{depth} R / I^{n}$, the last statement of the proposition is also clear.

In the case of a wonderful poset it is possible to express the depths of the powers directly in terms of invariants of $\Omega$ and $H$; cf. [DEP, §8] for the facts needed below.

(3.3.2) Corollary. Moreover, let $H$ be wonderful. Then:

(i) $\operatorname{depth} I^{n} \geq \operatorname{rk} H-\operatorname{rk} \Omega+1$,

(ii) $\operatorname{depth} R / I^{n} \geq \operatorname{rk} H-\operatorname{rk} \Omega$.

The bound in (ii) holds for the higher conormal modules $I^{n} / I^{n+1}$ as well.

Proof. As before it suffices to prove (i). Now $R / \Psi(h) R$ is a graded ordinal Hodge algebra on $H \backslash \Psi(h)$ and maximal chains in wonderful posets have the same number of elements. Therefore rk $H \backslash \Psi(h)=\mathrm{rk} H-\mathrm{rk} h+1$. Furthermore $H \backslash \Psi(h)$ is again wonderful. The result follows from well-known properties of wonderful posets: $(H \backslash \Psi(h)) R / \Psi(h) R$ contains an $R / \Psi(h) R$-regular sequence of length rk $H \backslash \Psi(h)$.

Even for wonderful posets the preceding inequalities may be strict for all values of $n$. Here is a simple instance: $R=K[X]$, with $X$ a $2 \times 2$ matrix of indeterminates, $H=\{$ all minors of $X\}$ and $\Omega=\left\{X_{11}, X_{12}\right.$, det $\left.X\right\}$. One has, however,

(3.3.3) Theorem. Let $R$ be a graded ordinal Hodge algebra over $B$ on a wonderful poset $H$. Let $\Omega \subset H$ be a straightening-closed ideal such that:

(i) The standard monomials occurring in the straightening relations of $R$ have at most two factors. 
(ii) With respect to the graduation of $R$, the elements of $\Omega$ have the maximal degree occurring among the elements of $H$.

Then min depth $R / I^{n}=\mathrm{rk} H-\mathrm{rk} \Omega$.

Proof. First we want to reduce the problem to the case in which $B$ is a field. Using the local description of depth, one has to determine the minimum of $\operatorname{depth}\left(R / I^{n}\right)_{P}$ over all $n$ and all prime ideals $P \supset H R$. Let $p=P \cap B$ and $\kappa(p)=(B / p B)_{p}$. Since $R / I^{n}$ is $B$-flat (in fact, $B$-free) one has

$$
\left.\operatorname{depth}\left(R / I^{n}\right)_{P}=\operatorname{depth}\left(\left(R / I^{n}\right) \otimes \kappa(p)\right)_{P / p R}\right)+\operatorname{depth} B_{p} .
$$

So it is obviously enough to show that $\operatorname{depth}\left(R / I^{n} \otimes \kappa(p)\right)=\operatorname{rk} H-\operatorname{rk} \Omega$ for every prime ideal $p$ of $B$. Observing that under the present circumstances the formation of $R / I^{n}$ commutes with the base change from $B$ to $\kappa(p)$, we may in fact assume that $B$ is a field. Then $P=H R$.

Let $S \subset R$ denote the $B$-subalgebra generated by the elements of $\Omega$. The fact that $\Omega$ is straightening-closed and condition (i) imply that every monomial in the elements of $\Omega$ is a linear combination of standard monomials consisting of factors from $\Omega$ only. Thus $S$ is a Hodge subalgebra of $R$ in a natural way. Letting $H^{*}$ and $\Omega^{*}$ denote the corresponding poset and ideal in $\operatorname{gr}_{I}(R)$, the Hodge $B$-subalgebra of $\operatorname{gr}_{I}(R)$ generated by $\Omega^{*}$ is naturally isomorphic to $S$ : the straightening relations for products $h k, h, k \in \Omega$, are homogeneous with respect to the filtration defined by the powers of $I$, and thus carry over unchanged to $\operatorname{gr}_{I}(R)$. Identifying these two algebras, we conclude that $S$ is a homomorphic image of the residue ring $\operatorname{gr}_{I}(R) / \mathrm{Hgr}_{I}(R)$. But, from general facts of Hodge algebras, one has $\operatorname{dim} S=\operatorname{dim} B+\operatorname{rk} \Omega$, cf. [BV, (5.10)] for example. Therefore

$$
\operatorname{dim} \operatorname{gr}_{I}(R) / H \operatorname{gr}_{I}(R) \leq \operatorname{dim} B+\mathrm{rk} \Omega=\operatorname{rk} \Omega
$$

We claim that under conditions (i) and (ii) equality takes place, since actually $\left.S \simeq \operatorname{gr}_{I}(R) / H \operatorname{gr}_{I}(R)\right)$. One has to show that $\left(H \operatorname{gr}_{I}(R)\right) \cap S=0$ and for this it suffices that every standard monomial in the standard representation of an element of $H \operatorname{gr}_{I}(R)$ contains a factor from $H^{*} \backslash \Omega^{*}$, equivalently, that every standard monomial $M$ appearing in a straightening relation of $\operatorname{gr}_{I}(R)$,

$$
h k=\sum b_{M} M
$$

with $h \in H^{*} \backslash \Omega^{*}$, contains at least one factor from $H^{*} \backslash \Omega^{*}$. Since this equation is homogeneous in the graded ring $\operatorname{gr}_{1}(R)$, a standard monomial $M$ can have at most one factor from $\Omega^{*}$. If $k \in H^{*} \backslash \Omega^{*}$, too, then every $M$ consists of factors from $H^{*} \backslash \Omega^{*}$ only. Otherwise $M$ must have two factors because of conditions (i) and (ii): the straightening relations of $\operatorname{gr}_{I}(R)$ are derived from those of $R$ by homogenization with respect to the filtration $\left\{I^{n}\right\}$, and if one of the factors is an element of maximal degree, then every monomial on the right-hand side must have exactly two factors. 
To complete the proof of the theorem, one observes that $\operatorname{gr}_{I}(R)$ is CohenMacaulay over a field $B$ and recalls the equality

$$
\min \operatorname{depth} R / I^{n}=\operatorname{dim} \operatorname{gr}_{I}(R)-l(I)
$$

whenever $\operatorname{gr}_{I}(R)$ is Cohen-Macaulay (cf., e.g., $\left.[\mathrm{EH}]\right)$. In the present context the analytic spread $l(I)$ is simply given by $\operatorname{dim} \operatorname{gr}_{I}(R) / H \mathrm{gr}_{I}(R)$.

\section{A STRAightening-CLOSED ZOO}

In this section we describe the most common appearances of straighteningclosed ideals. The list is certainly short of being complete. For the ring-theoretic properties to be discussed one should note that it is enough to consider the case in which the ring $B$ of coefficients is a field. All the ideals considered below, more precisely the residue class rings, associated graded rings and Rees algebras they define, are obtained for general $B$ from those over the integers $\mathbf{Z}$ by base change, and the latter are faithfully flat, in fact free Z-modules, since they are Hodge algebras. Cf. [Ma] for the property "Cohen-Macaulay", [Wa] for "Gorenstein", [BV, 3.C] for "reduced", "integral domain", and "normal".

4.1. Virtual maximal minors in determinantal rings. These ideals were introduced in (2.1.5). Here we want to restrict ourselves to the subclass given by

$$
I=\left(I_{s}(X \mid w)+I_{s+1}(X)\right) / I_{s+1}(X) \subset R=B[X] / I_{s+1}(X),
$$

where $X$ is an $m \times n$ generic matrix, $1 \leq s \leq \min \{m, n\}$ and $(X \mid w)$ denotes the submatrix consisting of the first $w$ columns of $X, s \leq w \leq n$. Note that the ordinary maximal minors are obtained when $s=\min \{m, n\}$ and $w=n$. The rings $R / I$ are determinantal rings in the sense of [BV] and therefore share the properties of being reduced, an integral domain, normal, or Cohen-Macaulay with $B$.

(4.1.1) Proposition. Let $I \subset R$ be the ideal of maximal minors just defined. Then:

(i) $\mathscr{R}(I)$ and $\operatorname{gr}_{I}(R)$ are graded ordinal Hodge algebras on wonderful posets.

(ii) $\operatorname{gr}_{I}(R)$ is $R / I$-torsionfree (equivalently, $I^{k}=I^{(k)}$ for all $k$ ), and

$$
\min \operatorname{depth} R / I^{k}=\min \{m, n\} s-1 \text {. }
$$

(iii) $\operatorname{gr}_{I}(R)$ is Cohen-Macaulay, reduced or a domain along with $B$.

(iv) $\operatorname{gr}_{I}(R)$ is Gorenstein if and only if $R$ is Gorenstein, i.e., if $B$ is Gorenstein and $s=\min \{m, n\}$ or $m=n$.

Suppose now that $B$ is a normal domain. Then:

(v) $\mathscr{R}(I)$ and $\operatorname{gr}_{I}(R)$ are normal domains.

(vi) $\mathrm{Cl}(\mathscr{R}(I)) \simeq \mathrm{Cl}(B) \oplus \mathbf{Z}^{r}$ and $\mathrm{Cl}\left(\operatorname{gr}_{I}(R)\right) \simeq \mathrm{Cl}(B) \oplus \mathbf{Z}^{u}$, where

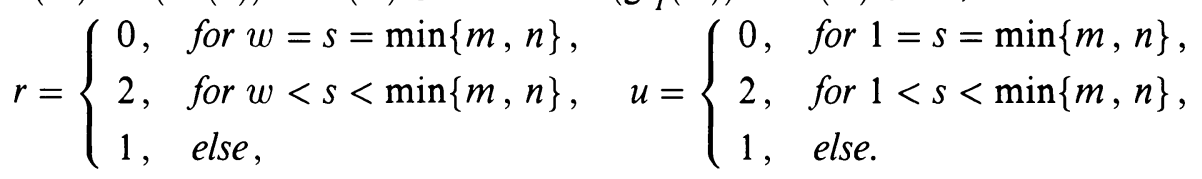


Proof. For $\operatorname{gr}_{I}(R)$ part (i) follows immediately from the results of subsection 2.2 , and for $\mathscr{R}(I)$ one applies [BV, (9.11)]. The first part of (ii) can be reduced to the case of a field $B$ by the same technique as employed in the proof (3.3.3), which, together with an easy computation of $\mathrm{rk} \Omega$, implies the second part of (ii). (Of course $\Omega$ denotes the poset ideal generating $I$.) By what has been said above, the assertion in (iii) regarding reducedness and integrality follows from (v). The Cohen-Macaulay part is covered by (i). For (iv) one again reduces the problem to a field of coefficients first. By (iii), $\operatorname{gr}_{I}(R)$ is a Cohen-Macaulay domain and [HSV 1, (3.7)] can be applied: $\omega_{G} \simeq \operatorname{gr}_{I}\left(\omega_{R}\right)$. In particular $\omega_{G}$ is cyclic if and only $\omega_{R}$ is cyclic.

(v) One may assume that $B$ is a field. Furthermore the integrality of $\operatorname{gr}_{I}(R)$ implies the normality of $\mathscr{R}(I)$. For $\operatorname{gr}_{I}(R)$ we use an inductive method which will also yield (vi). The crucial arguments are provided by the following lemma. (For a ring $S$, an ideal $J$, and an $S$-module $M$ we denote the length of a maximal $M$-sequence in $J$ by $\operatorname{grade}(J, M)$, and $\operatorname{grade} J=\operatorname{grade}(J, R)$.)

(4.1.2) Lemma. (a) If $s=1$, then $\operatorname{gr}_{I}(R) \simeq R$.

(b) If $s>1$, then

$$
\operatorname{gr}_{I}(R)\left[\left(x_{11}^{*}\right)^{-1}\right] \cong \operatorname{gr}_{J}(S)\left[X_{11}, \ldots, X_{1 n}, X_{21}, \ldots, X_{m 1}, X_{11}^{-1}\right],
$$

$S=B[Y], Y$ an $(m-1) \times(n-1)$ matrix of indeterminates and

$$
J=\left(I_{s-1}(Y \mid w-1)+I_{s}(Y)\right) / I_{s}(Y) .
$$

(c) Suppose that $s>1$. Then, $K$ denoting the ideal generated by the $x_{i j}^{*} \in$ $R / I, 1 \leq j \leq w$, one has

$$
\operatorname{grade} K \operatorname{gr}_{I}(R)=\min \operatorname{grade}\left(K, I^{i} / I^{i+1}\right) \geq 3 .
$$

Proof. (a) Both $\operatorname{gr}_{I}(R)$ and $R$ are residue class rings of $B[X]$ with respect to the assignments $X_{i j} \rightarrow x_{i j}^{*}$ and $X_{i j} \rightarrow x_{i j}$ respectively. In both cases the defining ideals are generated by the straightening relations, and these obviously coincide.

(b) In order to obtain the isomorphism, one performs elementary transformations on the matrix $X$ after the inversion of $X_{11}$-see [BV, (2.4)] for a prototype of the argument.

(c) Again one may assume that $B$ is a field. The equality claimed is elementary, and for the inequality one may replace $I^{i} / I^{i+1}$ by the factors $\bar{R}_{\omega}=$ $R /(\Psi(\omega) R+I)$ of its filtrations obtained in the proof of (2.3.3), $\omega$ running through the generators of $I$. This amounts to showing grade $K \bar{R}_{\omega} \geq 3$ for all $\omega$. We translate the present notations into those introduced in (2.1.5), using $\sqcup$ to denote the join operation in the lattice $\Delta(X)$ and $\widetilde{K}$ for the preimage of $K$ in $R$ :

$$
R / I=B[X] / I(X ; \varepsilon), \quad \varepsilon=\left\{\begin{array}{r}
{[1, \ldots, s \mid 1, \ldots, s-1, w+1]} \\
\text { for } w<n \\
{[1, \ldots, s-1 \mid 1, \ldots, s-1]} \\
\text { for } w=n
\end{array}\right.
$$




$$
\begin{aligned}
& \bar{R}_{\omega}=B[X] / I(X ; \zeta), \quad \zeta=\varepsilon \sqcup \omega, \\
& R / \widetilde{K}=\left\{\begin{array}{l}
B, \text { for } w=n, \\
B[X] / I(X ; \eta), \text { else, }
\end{array} \quad \eta=[1, \ldots, v \mid w+1, \ldots, w+v],\right. \\
& v=\min \{s, n-w\}, \\
& \bar{R}_{\omega} / K \bar{R}_{\omega}=\left\{\begin{array}{l}
B, \text { for } w=\eta, \\
B[X] / I(X, \vartheta), \text { else, } \quad \vartheta=\eta \sqcup \omega .
\end{array}\right.
\end{aligned}
$$

All these rings are Cohen-Macaulay, cf. [BV, (5.17)]. Therefore

$$
\text { grade } K \bar{R}_{\omega}=\operatorname{dim} \bar{R}_{\omega}-\operatorname{dim} \bar{R}_{\omega} / K \bar{R}_{\omega} \text {. }
$$

Let $\omega=\left[a_{1}, \ldots, a_{s} \mid b_{1}, \ldots, b_{s}\right]$ and observe for the inequalities to be discussed that $b_{1} \leq w-s+1, \ldots, b_{s} \leq w$.

We first dispose of the case in which $w=n$. Then

and, by $[\mathrm{BV},(5.12)]$,

$$
\zeta=\left[a_{1}, \ldots, a_{s-1} \mid b_{1}, \ldots, b_{s-1}\right], \quad R_{\omega} / K \bar{R}_{\omega}=B,
$$

$$
\begin{aligned}
\operatorname{grade} K \bar{R}_{\omega} & =\operatorname{dim} \bar{R}_{\omega}=(m+n)(s-1)-\sum_{i=1}^{s-1}\left(a_{i}+b_{i}\right)+s-1 \\
& \geq 2 \sum_{i=1}^{s-1}(s-i)+s-1=s^{2}-1 \geq 3 .
\end{aligned}
$$

Next let $w<n$ and $v=s$. Applying the same dimension formula, one gets

$$
\operatorname{grade} K \bar{R}_{\omega}=\sum_{i=1}^{s-1}\left(w+i-b_{i}\right)+(w+s)-(w+1) \geq s^{2}-1 \geq 3
$$

Finally suppose that $w<n, v<s$. Then $\vartheta=\left[a_{1}, \ldots, a_{v} \mid w+1, \ldots, w+v\right]$. We put $\tilde{\zeta}=\left[a_{1}, \ldots, a_{v} \mid b_{1}, \ldots, b_{v}\right]$. Obviously $\operatorname{rk} \tilde{\zeta}-\operatorname{rk} \zeta \geq s-v$ and $\operatorname{rk} \vartheta-$ $\operatorname{rk} \tilde{\zeta}=\sum_{i=1}^{v} w+i-b_{i} \geq v s$, altogether $\operatorname{grade} K \bar{R}_{\omega}=\operatorname{dim} \bar{R}_{\omega}-\operatorname{dim} \bar{R}_{\omega} / K \bar{R}_{\omega}=$ $\mathrm{rk} \vartheta-\mathrm{rk} \zeta \geq s-v+v s \geq 3$.

Continuing the proof of $(4.1 .1)(\mathrm{v})$ we observe that, for $s=1$, the normality of $\operatorname{gr}_{I}(R)$ follows from the normality of $R$ by (4.1.2)(a). Let $s>1$. Applying Serre's normality criterion it is sufficient to show that $\left(\operatorname{gr}_{I}(R)\right)_{P}$ is a regular local ring for all prime ideals $P$ of $\operatorname{gr}_{I}(R)$ such that $\operatorname{depth}\left(\operatorname{gr}_{I}(R)\right)_{P} \leq 1$. By $(4.1 .2)$ (c) $P$ cannot contain $K$. Thus there is an $x_{i j}^{*}, j \leq w$, such that $x_{i j}^{*} \notin P$. For symmetry reasons one may assume $x_{i j}^{*}=x_{11}^{*}$. Then $(4.1 .2)(\mathrm{b})$ reveals that $\left(\operatorname{gr}_{I}(R)\right)_{P}$ is a localization of a ring which, by induction, is normal. So $\left(\operatorname{gr}_{I}(R)\right)_{P}$ is normal, equivalently, regular.

The assertion on $\mathrm{Cl}(\mathscr{R}(I))$ in (vi) follows at once from (3.2.3): For every prime ideal $p$ of $B$ the extension $p R$ is even prime, for $R$ is a domain over a domain of coefficients; $\mathrm{Cl}\left(R_{B \backslash 0}\right)=0$ for $s=\min (m, n), \mathrm{Cl}\left(R_{B \backslash 0}\right)=\mathbf{Z}$ else $[\mathrm{BV},(8.4)]$. Furthermore the single minimal prime ideal 0 of $\operatorname{gr}_{I}(R)$ contracts to $I$, and $I$ has height 1 if and only if $w=s$. In order to compute $\mathrm{Cl}\left(\operatorname{gr}_{I}(R)\right)$ we need another lemma. 
(4.1.3) Lemma. (a) If $s=2$, then $x_{11}^{*}$ is the intersection of two height one primes $Q_{1}$ and $Q_{2}$ in $\operatorname{gr}_{I}(R)$, and $\mathrm{Zcl}\left(Q_{1}\right)=\mathbf{Z} \operatorname{cl}\left(Q_{2}\right) \cong \mathbf{Z}$.

(b) If $s>2, x_{11}^{*}$ is a prime element in $\operatorname{gr}_{I}(R)$.

Before showing (4.1.3) we complete the proof of (4.1.1). By (4.1.1)(iii) (which has been proved already) the extension $B \rightarrow \operatorname{gr}_{I}(R)$ is unramified in codimension one; thus we may invert $B \backslash 0$ and henceforth assume that $B$ is a field. Now induction on $s$ based on $(4.1 .2)(\mathrm{b}),(4.1 .3)(\mathrm{b})$, and Nagata's theorem reduces the case $s>2$ to the one in which $s=2$. If $s=2$ we can again use Nagata's theorem to obtain an exact sequence

$$
0 \rightarrow \mathbf{Z} \rightarrow \mathrm{Cl}\left(\operatorname{gr}_{I}(R)\right) \rightarrow \mathrm{Cl}\left(\operatorname{gr}_{J}(S)\right) \rightarrow 0
$$

from (4.1.2)(b) and (4.1.3)(a), hence we are left with the case $s=1$, settled by (4.1.2)(a) and [BV, (8.4)].

Proof of (4.1.3). Let $\mathrm{G}=\operatorname{gr}_{I}(R), x=x_{11}^{*}$. (a) We first show that $G / G x$ is reduced. It is enough to prove that the localization $(G x)_{P}$ is a radical ideal for all $P \in$ Ass $G / G x$. Because of $(4.1 .1)($ c),$P$ cannot contain all the elements $x_{i j}^{*}, j \leq w$. Invert $x_{i j}^{*}$ first. Then by $(4.1 .2)(\mathrm{b})(G x)_{P}$ is a prime ideal if $i=1$ or $j=1$. Otherwise

$$
x_{11}^{*}=x_{i 1}^{*} x_{1 j}^{*}\left(x_{i j}^{*}\right)^{-1}
$$

is a product of two prime elements in $G\left[\left(x_{i j}^{*}\right)^{-1}\right]$, more than needed to make $(G x)_{P}$ a radical ideal.

Since $x_{12}^{*} x_{21}^{*} \in G x, G x$ cannot be prime. By the argument just given, there is a single $Q_{1} \in$ Ass $G / G x$ such that $x_{12}^{*} \notin Q_{1}$ and a single $Q_{2} \in$ Ass $G / G x$ such that $x_{21}^{*} \notin Q_{2}$. Necessarily $Q_{1} \neq Q_{2}$. Suppose there is a third prime ideal $P \in$ Ass $G / G x$ and take $x_{i j}^{*}$ as above such that $x_{i j}^{*} \notin P$. Then the equation (*) implies that $x_{11}^{*} \in P^{(2)}$, a contradiction to the fact that $(G x)_{P}$ is a radical ideal.

In order to prove that $\mathrm{Z} \operatorname{cl}\left(Q_{1}\right) \cong \mathbf{Z}$ one uses the same "unit trick" as in $[\mathrm{BV}$, p. 94].

(b) We first show that the extension of $G x$ to $G\left[\left(x_{i j}^{*}\right)^{-1}\right]$ is prime for all $i, j, j \leq w$. For $i=1$ or $j=1$ this follows as above. In the remaining case we have

$$
x=x_{11}^{*}= \pm y_{i j}^{*}+X_{i 1} X_{i j}^{-1} X_{1 j},
$$

identifying the rings in (an appropriate version of) (4.1.2) (b). Now $y_{i j}^{*}$ is not a zero-divisor in $\operatorname{gr}_{I}(S)$, thus $y_{i j}^{*}, X_{i 1} X_{i j}^{-1}$ is a regular sequence in

$$
\operatorname{gr}_{I}(S)\left[X_{11}, \ldots, \widehat{X}_{i j}, \ldots, X_{1 n}, X_{21}, \ldots, X_{m 1}, X_{11}^{-1}\right] \text {. }
$$

Adding the indeterminate $X_{i j}$ we conclude that $x$ indeed generates a prime ideal in $G\left[\left(x_{i j}^{*}\right)^{-1}\right]$.

As in (a) $G x$ must be a radical ideal. Let $Q \in$ Ass $G / G x$. Then there is an $x_{i j}^{*} \notin Q, j \leq w$, and $Q$ is the single element of Ass $G / G x$ not containing 
$x_{i j}^{*}$. Suppose there is a second associated prime $P$ and choose $x_{u v}^{*}$ accordingly. Then $x_{u v}^{*} \in Q$ and $x_{u v}^{*} \in x G\left[\left(x_{i j}^{*}\right)^{-1}\right]$. To put it differently: $\left(x_{i j}\right)^{e} x_{u v}^{*} \in G x$ for sufficiently high $e$. For reasons of homogeneity we can replace $G$ by its component $R / I$ and obtain a contradiction to the fact that $x_{11}$ is a prime element in $R / I$, cf. [BV, (12.6)] or [HE].

4.2. Maximal minors fixing a submatrix. This example has been briefly mentioned in (2.1.4). As before, $X$ stands for an $m \times n$ matrix of indeterminates over a commutative ring $B$. We assume $m \leq n$ and fix an integer $r$, $1 \leq r<m$; note that the (trivial) case $m=r$ is covered by (4.1.1). The poset ideal $\Gamma_{r}(X)$ in question (cf. (2.1.4)) is the intersection of the poset ideal of all minors of $X$ involving the submatrix $(X \mid r)$ consisting of the first $r$ columns of $X$ and the poset ideal of all maximal minors of $X$. Therefore, by a standard principle in Hodge algebra theory,

$$
J_{r}(X)=I_{r}(X \mid r) \cap I_{n}(X)
$$

where $J_{r}(X)=\Gamma_{r}(X) R$ and $R=B[X]$. In particular, if $B$ is reduced (resp. a domain) then $J_{r}=J_{r}(X)$ is a radical ideal (resp. Ass $R / J_{r}=\left\{I_{r}(X \mid r), I_{n}(X)\right\}$ and $J_{r}$ is generically a complete intersection).

(4.2.1) Proposition. The following statements are equivalent:

(i) $J_{r}$ is of linear type, i.e., $\mathscr{S}(I) \simeq \mathscr{R}(I)$.

(ii) $m=n+1$ or $n=r+1$.

This follows very easily from (2.2.2).

(4.2.2) Proposition. Let $B$ be a noetherian ring. Set $R=B[X]$ and $I=J_{r}$. Then:

(i) $\mathscr{R}(I)$ and $\operatorname{gr}_{I}(R)$ are graded ordinal Hodge algebras on wonderful posets.

(ii) $\operatorname{gr}_{I}(R)$ is $R / I$-torsionfree; equivalently, $I^{k}=I^{(k)}$ for all $k$.

(iii) If $B$ is Cohen-Macaulay, $\mathscr{R}(I)$ and $\operatorname{gr}_{I}(R)$ are Cohen-Macaulay. If $B$ is reduced, $\operatorname{gr}_{I}(R)$ is reduced, too.

(iv) If $B$ is Gorenstein, $\operatorname{gr}_{I}(R)$ is Gorenstein if and only if $n=(m+r) / 2$.

(v) If $B$ is a normal domain, $\mathscr{R}(I)$ is normal and $\mathrm{Cl}(\mathscr{R}(I)) \simeq \mathrm{Cl}(B) \oplus \mathbf{Z}^{2}$.

Proof. As in the proof of (4.1.1) one reduces everything to the case of a field $B$ of coefficients first. For (i) and (iii) one argues as for (4.1.1). For the rest of the statements we can now even use that $R / I$ has finite projective dimension over $B[X]-B$ is a field. The torsion-freeness of $\operatorname{gr}_{I}(R)$ follows immediately from (3.1.2), and for the Gorenstein property and (v) one applies (3.2.4) and (3.2.3) respectively.

See [AS 1] and [AS 2] for a more detailed investigation of some special classes of ideals of minors fixing a submatrix.

The last result concerns the arithmetical rank ara $J_{r}$, i.e., the minimal number of elements that suffice to generate $J_{r}$ up to radical. By a well-known fact of 
the combinatorics of determinants, it is easier to treat the Grassmannian $G(X)$ first rather than the polynomial ring $B[X]$, where, with the notation introduced in (2.1.6), $G(X)$ is the $B$-subalgebra of $B[X]$ generated by the maximal minors of $X$. For example, since $G(X)$ is a symmetric Hodge algebra, one has [BV, $(5.22)]$

$$
\operatorname{ara} \Gamma_{r}(X) G(X)=\operatorname{rk} \Gamma_{r}(X)=(m-n)(n-r)+1 .
$$

In general it is not clear how the arithmetical rank behaves under an extension of Hodge algebras. However, with the extra requirement of characteristic zero, a cohomological argument, going back to Hochster [BV, (7.12)], works to give the exact value of ara $J_{r}$.

First, we have the following lemma, regardless of any assumption on the characteristic.

(4.2.4) Lemma. Let $T(X) \subset G(X)$ denote the B-subalgebra generated by the elements of $\Gamma_{r}(X)$. Then $T(X)$ is a direct $T(X)$-summand of $G(X)$.

Proof. Let $C$ be the $B$-submodule of $G(X)$ generated by the standard monomials having a factor outside $T(X)$. Clearly $C$ is a direct $B$-summand of $G(X)$ with complement $T(X)$ and it remains to see that it is also a $T(X)$-submodule of $G(X)$. For this it suffices to show that, given a standard monomial $\mu \in C$ and an element $\delta \in \Gamma_{r}(X)$, the standard representation $\mu \delta=\sum_{\nu} a_{\nu} \nu$ is such that every monomial $\nu$ contains a factor not belonging to $T(X)$. Moreover, since the standard representation is obtained by repeated applications of the straightening relations, the argument boils down to showing that, if $\xi \eta=\sum_{\nu} a_{\nu} \nu$ is a straightening in $G(X)$ such that some monomial $\nu$ has two factors from $T(X)$, then all remaining monomials have two factors from $T(X)$ as well. But the latter follows easily from the statements (i) and (ii) in (2.1.3) on the straightening law in $B[X]$.

(4.2.5) Theorem. If there is a homomorphism $B \rightarrow K$, with $K$ a field of characteristic zero, then ara $J_{r}=(m-n)(n-r)+1$.

Proof. Note that ara $J_{r} \leq \operatorname{ara} \Gamma_{r}(X) G(X)=(m-n)(n-r)+1$. Therefore one can assume that $B=K$. By (4.2.4), $T(X)$ is a direct $T(X)$-summand of $G(X)$ and $G(X)$ is a direct $G[X]$-summand of $K[X]$, cf. [BV, 7.D]; altogether $T(X)$ is a direct $T(X)$-summand of $K[X] . \Gamma_{r}(X) T(X)$ is the irrelevant maximal ideal of $T(X)$, and arguing with cohomological dimension one shows easily that $\operatorname{ara} J_{r} \geq \operatorname{dim} T(X)=\operatorname{rk} \Gamma_{r}(X)$, cf. [BV, (7.12)] for the details.

4.3. The two by two minors as an extended Rees algebra. The representation discussed in this topic depends on example (2.1.7). Referring to the notation there, one has

(4.3.1) Lemma. Let $R=B[X, Y]$ and $I=\Omega R$. Set $\widetilde{H}=H^{*} \cup\left\{t^{-1}\right\}$, where, as usual, $H^{*}$ stands for the poset of leading forms and $t^{-1}$ is decreed the greatest element of $\widetilde{H}$. Then

(i) $R\left[I t, t^{-1}\right]$ is a graded ordinal Hodge algebra on $\widetilde{H}$ over $B$. 
(ii) $R\left[I t, t^{-1}\right] \simeq B[T] / I_{2}(T)$ as graded Hodge algebras, where $T$ is an $(m+1) \times(n+1)$ matrix of indeterminates over $B$ and the isomorphism is defined by

$$
\begin{array}{ll}
T_{i j} \mapsto X_{i} Y_{j} t, & i=1, \ldots, m, j=1, \ldots, n, \\
T_{m+1 j} \mapsto Y_{j}, & T_{i n+1} \mapsto X_{i}, \quad T_{m+1 n+1} \mapsto t^{-1} .
\end{array}
$$

Proof. (i) By a standard argument [DEP], $R\left[I t, t^{-1}\right]$ is a graded ordinal Hodge algebra on $H^{*}$ over $B\left[t^{-1}\right]$, hence it is a graded ordinal Hodge algebra on $\widetilde{H}$ over $B$. (ii) The defined assignment induces a (surjective) homomorphism since the $2 \times 2$ minors of the matrix

$$
\left(\begin{array}{cccc}
X_{1} Y_{1} t & \ldots & X_{1} Y_{n} t & X_{1} \\
\vdots & \ddots & \vdots & \vdots \\
X_{m} Y_{1} t & \ldots & X_{m} Y_{n} t & X_{m} \\
Y_{1} & \ldots & Y_{n} & t^{-1}
\end{array}\right)
$$

vanish under this assignment. On the other hand, the relatins so-obtained are exactly the straightening relations of $R\left[I t, t^{-1}\right]$ over $B$. Therefore, the homomorphism is injective.

As a consequence of the above presentation, one recovers, quite independently, some of the main classical results about $2 \times 2$ generic minors. We collect some of these in the following proposition.

(4.3.2) Proposition. Let $B$ be a noetherian ring, and let $T$ be a generic $(m+1) \times(n+1)$ matrix over $B$. Then:

(i) $\operatorname{dim} B[T] / I_{2}(T)=\operatorname{dim} B+m+n+1$.

(ii) [HE] If $B$ is reduced, $B[T] / I_{2}(T)$ is reduced.

(iii) [Sh] If $B$ is a domain, $I_{2}(T)$ is a prime ideal.

(iv) [Sh] If $B$ is Cohen-Macaulay, $B[T] / I_{2}(T)$ is Cohen-Macaulay.

(iv) $[\mathrm{HE}]$ If $B$ is a normal domain, $B[T] / I_{2}(T)$ is a normal domain.

(vi) [Sv] If $B$ is Gorenstein, $B[T] / I_{2}(T)$ is Gorenstein if and only if $m=n$.

Proof. (i) follows from $\operatorname{dim} R\left[I t, t^{-1}\right]=\operatorname{dim} R+1$. (ii) and (iii) are immediate from the presentation in (4.3.1)(ii). (iii) The Cohen-Macaulay property of $B[T] / I_{2}(T)$ results from (2.2.5)(ii) applied to the setup of (2.1.7)-note the poset there is wonderful. Referring to the notation of (2.1.7), one has an exact sequence

$$
0 \rightarrow R / I \rightarrow R /(X) R \oplus R /(Y) R \rightarrow R /(X, Y) R \rightarrow 0 .
$$

Therefore $R / I$ has finite homological dimension over $R$. Applying (3.2.1) one obtains that $\mathscr{R}(I)$ is normal. Now it is well known that $R\left[I t, t^{-1}\right]$ is normal if $\mathscr{R}(I)$ is normal (cf., e.g., [HSV 2, (2.1.2)]). (v) In a vein similar to (iv), this follows from (3.2.4). 


\section{REFERENCES}

[AS 1] J. F. Andrade and A. Simis, On ideals of minors fixing a submatrix, J. Algebra 102 (1986), 246-259.

[AS 2] _ Free resolutions of certain codimension three perfect radical ideals, Arch. Math. $\mathbf{5 3}$ (1989), 448-460.

[BV] W. Bruns and U. Vetter, Determinantal rings, Lecture Notes in Math., vol. 1327 (Subseries: IMPA, Rio de Janeiro), Springer, Berlin, Heidelberg, and New York, 1988.

[DEP] C. De Concini, D. Eisenbud and C. Procesi, Hodge algebras, Astérisque 91 (1982).

[EH] D. Eisenbud and C. Huneke, Cohen-Macaulay Rees algebras and their specializations, J. Algebra 81 (1983), 202-224.

[Ha] R. Hartshorne, Cohomological dimension of algebraic varieties, Ann. of Math. 88 (1968), 403-450.

[HE] M. Hochster and J. Eagon, Cohen-Macaulay rings, invariant theory and the generic perfection of determinantal loci, Amer. J. Math. 93 (1971), 1020-1058.

[HSV 1] J. Herzog, A. Simis and W. Vasconcelos, On the canonical module of the Rees algebra and the associated graded ring of an ideal, J. Algebra 105 (1987), 285-302.

[HSV 2] _ - The arithmetic of normal Rees algebras, preprint.

[Hu 1] C. Huneke, On the symmetric and Rees algebra of an ideal generated by a $d$-sequence, J. Algebra 62 (1980), 268-275.

[Hu 2] _ _ Powers of ideals generated by weak d-sequences, J. Algebra 68 (1981), 471-509.

[HuSV] C. Huneke, A. Simis and W. Vasconcelos, Reduced normal cones are domains, Contemp. Math. (to appear).

[Ma] H. Matsumura, Commutative algebra, 2nd ed., Benjamin/Cummings, Reading, Mass., 1980.

[Sh] D. W. Sharpe, On certain polynomial ideals defined by matrices, Quart. J. Math. Oxford Ser. 15 (1964), 155-175.

[ST] A. Simis and N. V. Trung, The divisor class group of ordinary and symbolic blow-ups, Math. Z. 198 (1988), 479-491.

[Sv] T. Svanes, Coherent cohomology on Schubert subschemes of flag schemes and applications, Adv. in Math. 14 (1974), 369-453.

Universität Osnabrück, Abt. Vechta, Naturwiss., FB Mathematik, Driverstrasse 22, D-2848 VeCHTA, GERMANY

Universidade Federal da Bahia, Instituto de Matemática, Av.Ademar de Barros, S/N, 40210 Salvador, BaHIA, BRAZIL

Institute of Mathematics, Box 631 Bô Hô, Hanol, Vietnam 